\title{
Can we know the global structure of spacetime? ${ }^{\star}$
}

\author{
John Byron Manchak
}

\begin{abstract}
Here, we briefly review the notion of observational indistinguishability within the context of classical general relativity. We settle a conjecture given by Malament (1977) concerning the subject and then strengthen the result considerably. The upshot is this: There seems to be a robust sense in which the global structure of every cosmological model is underdetermined.
\end{abstract}

Key words: Epistemology, Observational Indistinguishability, Underdetermination, General Relativity, Cosmology

\section{Introduction}

Here, we consider some epistemological issues arising within the context of the general theory of relativity. The principal thesis to emerge is this: It appears to follow from relativity theory itself that we can know very little about the global structure of spacetime.

More specifically, our goal is to show that (1) excluding certain pathological examples, every cosmological model of our universe is empirically underdetermined; no amount of observational data we could ever (even in principle) accumulate, can force one and only one cosmological model upon us. Additionally, we also claim that (2) even if one assumes a principle of uniformity - that the physical laws we determine locally are applicable throughout the universe - these general epistemological difficulties remain.

We begin with a pair of general observations to frame the technical results below. First, in discussions of underdetermination within the philosophy of

\footnotetext{
‡ I wish to thank Jeffrey Barrett, John Earman, Robert Geroch, Clark Glymour, John Norton, and Kyle Stanford for valuable discussions on this topic. I am especially grateful to David Malament for his help and encouragement.
} 
science, one sometimes encounters assertions of the following kind: If we are willing to sufficiently revise our background physical theories, then no amount of evidence will ever compel us to embrace a particular scientific claim. However, it is absolutely imperative to note that there is a different sort of assertion altogether which states that similar epistemological predicaments may be inherited without renouncing or revising our best physical theories. Of course, it is this latter type of assertion that concerns us in this paper.

Second, a discussion of observationally indistinguishable cosmological models is sometimes used to buttress some broad philosophical stance. ${ }^{1}$ We are certainly interested in the implications of our work for these extensive projects. But we want to make it very clear, however, that we have no special stake in any one of these more general positions. The motivations for this paper have primarily stemmed from simply considering the observer's epistemological situation within our best large-scale physical theory. We feel it is of some interest that general relativity is the sort of physical theory that allows for a wide variety of cosmological models but that, due to structure internal to the theory itself, does not allow us to determine which of these models best represents our universe.

\section{Background structure}

We begin with a few preliminaries concerning the relevant background formalism of general relativity. ${ }^{2}$ A relativistic spacetime is a pair of mathematical objects $\left(M, g_{a b}\right) . M$ is a connected four-dimensional manifold (without boundary) that is smooth (infinitely differentiable). Here, $g_{a b}$ is a smooth, non-degenerate, pseudo-Riemannian metric of Lorentz signature $(+,-,-,-)$ defined on $M$. Each point in the manifold represents an "event" in spacetime. For each point $p$ in the manifold, the metric assigns a light cone structure in the tangent space $M_{p}$. Any tangent vector $\xi^{a}$ in $M_{p}$ will be timelike (if $g_{a b} \xi^{a} \xi^{b}>0$ ), null (if $g_{a b} \xi^{a} \xi^{b}=0$ ), or spacelike (if $g_{a b} \xi^{a} \xi^{b}<0$ ). Null vectors create the "cone" structure. Timelike vectors are inside the cone while spacelike vectors are outside. A time orientable spacetime is one that has a continuous timelike vector field on $M$. A time orientable spacetime allows us to distinguish between the future and past lobes of the light cone. In what follows, it is assumed that spacetimes are time orientable.

1 For example, see Glymour (1980), van Fraassen (1980), Sklar (1985), Earman (1993), Leplin (1997), Stanford (2006), and Norton (2008).

2 The reader is encouraged to consult Hawking \& Ellis (1973) and Wald (1984) for details. An outstanding (and less technical) survey of the global structure of spacetime is given by Geroch \& Horowitz (1979). 
For some interval $I \subseteq \mathbb{R}$, a smooth curve $\gamma: I \rightarrow M$ is timelike if the tangent vector $\xi^{a}$ at each point in $\gamma[I]$ is timelike. Similarly, a curve is null (respectively, spacelike) if its tangent vector at each point is null (respectively, spacelike). A timelike curve is future-directed if its tangent vector at each point lies in the future lobe of the light cone. For any two points $p, q \in M, q$ is to the timelike future of $p$ (written $p<<q$ ) if there exists a timelike, future-directed curve $\gamma$ from $p$ to $q$. A future-directed curve from $p$ to $q$ that is either timelike or null indicates that $q$ is to the causal future of $p$ (written $p<q$ ). These relations allow us to define the following sets of points in $M: I^{-}(p)=\{q: q<<p\}$, $I^{+}(p)=\{q: p<<q\}, J^{-}(p)=\{q: q<p\}$, and $J^{+}(p)=\{q: p<q\}$. The set $I^{-}(p)$ will be used extensively throughout the paper and is called the observational past of $p$. This set represents those points which can possibly be observed from $p .{ }^{3}$

Two spacetimes $\left(M, g_{a b}\right)$ and $\left(M^{\prime}, g_{a b}^{\prime}\right)$ are isometric if there is a diffeomorphism $\phi: M \rightarrow M^{\prime}$ such that $\phi_{*}\left(g_{a b}\right)=g_{a b}^{\prime}$. For ease of presentation, we will sometimes say that two manifolds $M$ and $M^{\prime}$ are isometric when it is clear which metrics are associated with $M$ and $M^{\prime}$. Two spacetimes $\left(M, g_{a b}\right)$ and $\left(M^{\prime}, g_{a b}^{\prime}\right)$ are locally isometric if, for each point $p \in M$, there is an open neighborhood $O$ of $p$ and an open subset $O^{\prime}$ of $M^{\prime}$ such that $O$ and $O^{\prime}$ are isometric, and, correspondingly, with the roles of $\left(M, g_{a b}\right)$ and $\left(M^{\prime}, g_{a b}^{\prime}\right)$ interchanged.

Finally, to facilitate the proof of the theorem below, we note the following lemma.

Lemma. Let $\left(M, g_{a b}\right)$ be any spacetime and let $p$ be any point in $M$. If $I^{-}(p) \subset M$ then $\overline{I^{-}(p)} \subset M .^{4}$

Proof. Assume $I^{-}(p) \subset M$. So there exists an $s \in M$ such that $s \notin I^{-}(p)$. Let $r$ be any point in $I^{+}(s)$. So $s \in I^{-}(r)$. But, we know that for any points $p, r, s \in M$, if $r \in \overline{I^{-}(p)}$ and $s \in I^{-}(r)$, then $s \in I^{-}(p)$. Hence, $r \notin \overline{I^{-}(p)}$. So $\overline{I^{-}(p)} \subset M$.

\section{Observational indistinguishability}

Now we consider what it would mean to say that two spacetimes are observational indistinguishable. The development of this subject can be traced back to Clark Glymour $(1972,1977)$ and David Malament (1977) and the reader is invited to study their papers. In what follows, we will restrict our atten-

3 One uses the set $I^{-}(p)$ instead of $J^{-}(p)$ to represent the "observational past" of $p$ for reasons of mathematical convenience.

4 Here, $\subset$ should be interpreted as "proper subset". 
tion to Malament's somewhat weaker (asymmetric) definition. ${ }^{5}$ We have the following.

Definition. Let $\left(M, g_{a b}\right)$ and $\left(M^{\prime}, g_{a b}^{\prime}\right)$ be spacetimes. We say $\left(M, g_{a b}\right)$ is $o b$ servationally indistinguishable from $\left(M^{\prime}, g_{a b}^{\prime}\right)$ if, for every point $p \in M$, there is a point $p^{\prime} \in M^{\prime}$ such that $I^{-}(p)$ and $I^{-}\left(p^{\prime}\right)$ are isometric.

So, given that a spacetime $\left(M, g_{a b}\right)$ is observationally indistinguishable from another spacetime $\left(M^{\prime}, g_{a b}^{\prime}\right)$, an observer at any point in $M$ cannot know which of the two spacetimes she inhabits. No amount of observational data she could ever, even in principle, accumulate, allow her to distinguish between the models $\left(M, g_{a b}\right)$ and $\left(M^{\prime}, g_{a b}^{\prime}\right)$. We are now prepared to consider an open question concerning observationally indistinguishable spacetimes.

Consider spacetimes $\left(M, g_{a b}\right)$ with the property that for some point $p$ in $M$, $I^{-}(p)=M$. Such spacetimes necessarily contain closed timelike curves and, in addition, have the property that all of spacetime may be observed from one point. We will call these spacetimes causally bizarre. Malament (1977) has conjectured that only causally bizarre spacetimes do not have a distinct (non-isometric) observationally indistinguishable counterpart.

Conjecture. (Malament) Let $\left(M, g_{a b}\right)$ be any spacetime which is not causally bizarre. There exists another spacetime $\left(M^{\prime}, g_{a b}^{\prime}\right)$ (one that is not isometric to $\left.\left(M, g_{a b}\right)\right)$ such that $\left(M, g_{a b}\right)$ is observationally indistinguishable from $\left(M^{\prime}, g_{a b}^{\prime}\right)$.

Malament's argument sketch in support of such a claim has been dubbed the "clothesline construction" and may be summarized as follows. ${ }^{6}$ Let $\left\{p_{i}\right\}$ be a countable sequence of points in $M$ such that $\bigcup\left\{I^{-}\left(p_{i}\right)\right\}=M .^{7}$ Like a "clothesline", it would seem that these $I^{-}\left(p_{i}\right)$ can be "strung out" with an appropriately chosen "spacetime filler" such that they are isometrically embedded (disjointly) in some other spacetime $\left(M^{\prime}, g_{a b}^{\prime}\right)$. The spacetime filler in between the embedded $I^{-}\left(p_{i}\right)$ is subject only to the constraint that the metric $g_{a b}^{\prime}$ be smooth. With great flexibility, then, it is possible to choose the filler so that $\left(M, g_{a b}\right)$ is not isometric to $\left(M^{\prime}, g_{a b}^{\prime}\right)$. But, because of the way $\left(M^{\prime}, g_{a b}^{\prime}\right)$ was constructed, every point $p \in M$ will be in some $I^{-}\left(p_{i}\right)$

5 What we simply call "observational indistinguishability" Malament calls "weak observational indistinguishability" to avoid confusion with Glymour's stronger (symmetric) formulation. For a discussion of the relationship between the definitions of Glymour and Malament and for numerous examples of observationally indistinguishable spacetimes, see Malament (1977).

6 As an argument sketch, we have intentionally left some of the steps somewhat imprecise. The reader is encouraged to consult the theorem below for a detailed proof of the claim.

7 Such a countable set of points exists in any spacetime. See Malament (1977, p. 80). 
that is isometrically embedded in $\left(M^{\prime}, g_{a b}^{\prime}\right)$. So, $\left(M, g_{a b}\right)$ is observationally indistinguishable from $\left(M^{\prime}, g_{a b}^{\prime}\right)$.

Although it seemed likely, it was not certain that Malament's intuitive argument sketch would eventually materialize into an honest proof. But, as we will shortly see, the conjecture is indeed true. So, there is a sense in which, excluding causally bizarre spacetimes, every observer in every spacetime inherits a serious epistemological predicament.

\section{An underdetermination theorem}

Of course, Malament's claim considers arbitrary spacetimes - no attempt is made to differentiate between cosmological models which are, in some sense, "physically reasonable" and those which are not. Thus, there is room to argue that there may not be any significant underdetermination at work after all; if the cosmologist takes seriously only "physically reasonable" spacetimes, it may be that her epistemological plight vanishes entirely. Here we provide a response to this line of reasoning.

If one is interested in realistic cosmological models, one may restrict one's attention to spacetimes satisfying any number of constraints. For example, Einstein's equation along with some reasonable matter source is often imposed to rule out seemingly artificial models. ${ }^{8}$ The precise formulation of Einstein's equation need not concern us here. What is important to note, however, is that it, along with many of the other conditions usually considered, are "local" in the sense that they are conserved under local isometries.

Formally, we say that a condition $C$ on a spacetime is local if, given any two locally isometric spacetimes $\left(M, g_{a b}\right)$ and $\left(M^{\prime}, g_{a b}^{\prime}\right),\left(M, g_{a b}\right)$ satisfies $C$ if and only if $\left(M^{\prime}, g_{a b}^{\prime}\right)$ satisfies $C$. We now strengthen Malament's claim considerably by showing that it holds even under the imposition of any local constraints on spacetime. We have the following.

Theorem. Let $\left(M, g_{a b}\right)$ be any spacetime which is not causally bizarre satisfying any set $\mathfrak{C}$ of local conditions. There exists another spacetime $\left(M^{\prime}, g_{a b}^{\prime}\right)$ (one that is not isometric to $\left.\left(M, g_{a b}\right)\right)$ such that (i) $\left(M^{\prime}, g_{a b}^{\prime}\right)$ satisfies the set $\mathfrak{C}$ of local conditions and (ii) $\left(M, g_{a b}\right)$ is observationally indistinguishable from $\left(M^{\prime}, g_{a b}^{\prime}\right)$.

Proof. Let $\left(M, g_{a b}\right)$ be any spacetime which is not causally bizarre satisfying any set $\mathfrak{C}$ of local conditions. Let $\left\{p_{i}\right\}$ be a countable sequence of points in $M$

8 The interested reader can consult Hawking \& Ellis (1973) or Wald (1984) for a formal discussion of Einstein's equation. 
such that $\bigcup\left\{I^{-}\left(p_{i}\right)\right\}=M$. By assumption and the lemma above, we know that for all $i, \overline{I^{-}\left(p_{i}\right)} \neq M$. So, associate with each point $p_{i}$ a point $q_{i} \notin \overline{I^{-}\left(p_{i}\right)}$ and neighborhood $O_{i}$ of $q_{i}$ such that $O_{i} \cap I^{-}\left(p_{i}\right)=\varnothing$. By the Hausdorff condition, for all $i$ the points $q_{i}$ and $q_{i+1}$ are such that we can make their associated neighborhoods $O_{i}$ and $O_{i+1}$ disjoint.

Now, let $K_{i}^{+}$be a closed three-dimensional spacelike surface such that $K_{i}^{+} \subset$ $O_{i}$. Similarly, let $K_{i}^{-}$be a closed three-dimensional spacelike surface such that $K_{i}^{-} \subset O_{i}$ and $K_{i}^{+} \cap K_{i}^{-}=\varnothing$. It is important to note that, by construction, $K_{i}^{+} \cap K_{i+1}^{-}=\varnothing$ for all $i$.

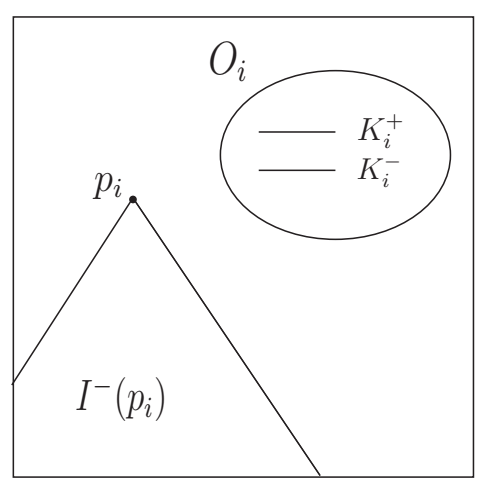

$\left(M(i, \alpha), g_{a b}\right)$

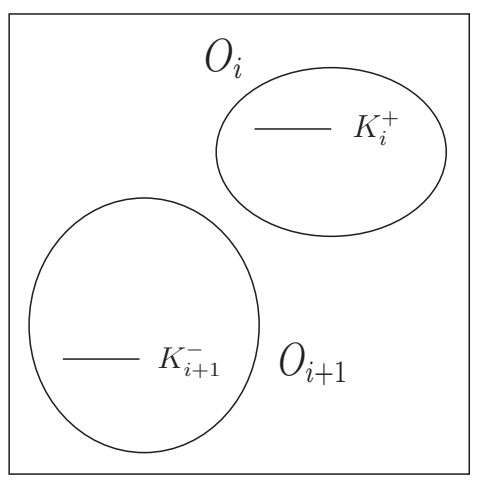

$\left(M(i, \beta), g_{a b}\right)$

Fig. 1. The spacetimes $\left(M(i, \alpha), g_{a b}\right)$ (for all $i \neq 1$ ) and $\left(M(i, \beta), g_{a b}\right)$ (for all $\left.i\right)$. Note that $\left(M(1, \alpha), g_{a b}\right)$ (not shown) is constructed without removing the set $K_{1}^{-}$.

Now, for each $i \neq 1$, let $M(i, \alpha)$ be the manifold $M-\left(K_{i}^{+} \cup K_{i}^{-}\right)$. Let $M(1, \alpha)=M-K_{1}^{+}$. Next, for all $i$ let $M(i, \beta)$ be the manifold $M-\left(K_{i}^{+} \cup K_{i+1}^{-}\right)$. Consider the spacetimes $\left(M(i, \alpha), g_{a b}\right)$ and $\left(M(i, \beta), g_{a b}\right)$ (see figure 1$)$. We make the following identifications: For all $i$, we identify the upper edge of $K_{i}^{+}$in $\left(M(i, \alpha), g_{a b}\right)$ with the lower edge of $K_{i}^{+}$in $\left(M(i, \beta), g_{a b}\right)$ (excluding the boundary points $\left.{ }^{9}\right)$ and the lower edge of $K_{i}^{+}$in $\left(M(i, \alpha), g_{a b}\right)$ with the upper edge of $K_{i}^{+}$in $\left(M(i, \beta), g_{a b}\right)$. Similarly, we identify the upper edge of $K_{i+1}^{-}$in $\left(M(i, \beta), g_{a b}\right)$ with the lower edge of $K_{i+1}^{-}$in $\left(M(i+1, \alpha), g_{a b}\right)$ and vice versa. ${ }^{10}$ The identifications produce a "chain" in which $\left(M(1, \alpha), g_{a b}\right)$ is attached to $\left(M(1, \beta), g_{a b}\right)$ which is attached to $\left(M(2, \alpha), g_{a b}\right)$ which is attached to $\left(M(2, \beta), g_{a b}\right)$ and so on (see figure 2$)$. By definition, this resulting "chain" is a spacetime - call it $\left(M^{\prime}, g_{a b}^{\prime}\right)$. We now show that $\left(M, g_{a b}\right)$ and $\left(M^{\prime}, g_{a b}^{\prime}\right)$ satisfy properties (i) and (ii).

$\overline{9}$ A 2-sphere makes up the boundary of the excised 3-disk. After the identifications, this 2-sphere is necessarily "left out" of the resulting spacetime. See Hawking \& Ellis (1973, p. 58-59) for further discussion.

${ }^{10}$ For other examples of this type of construction (by which one produces a spacetime by "cutting" and "pasting" together regions of other spacetimes), see Hawking \& Ellis (1973, p. 58-59) and Geroch (1977, p. 89-90). A short discussion of the procedure can be found in Geroch \& Horowitz (1979, p. 220-221). 


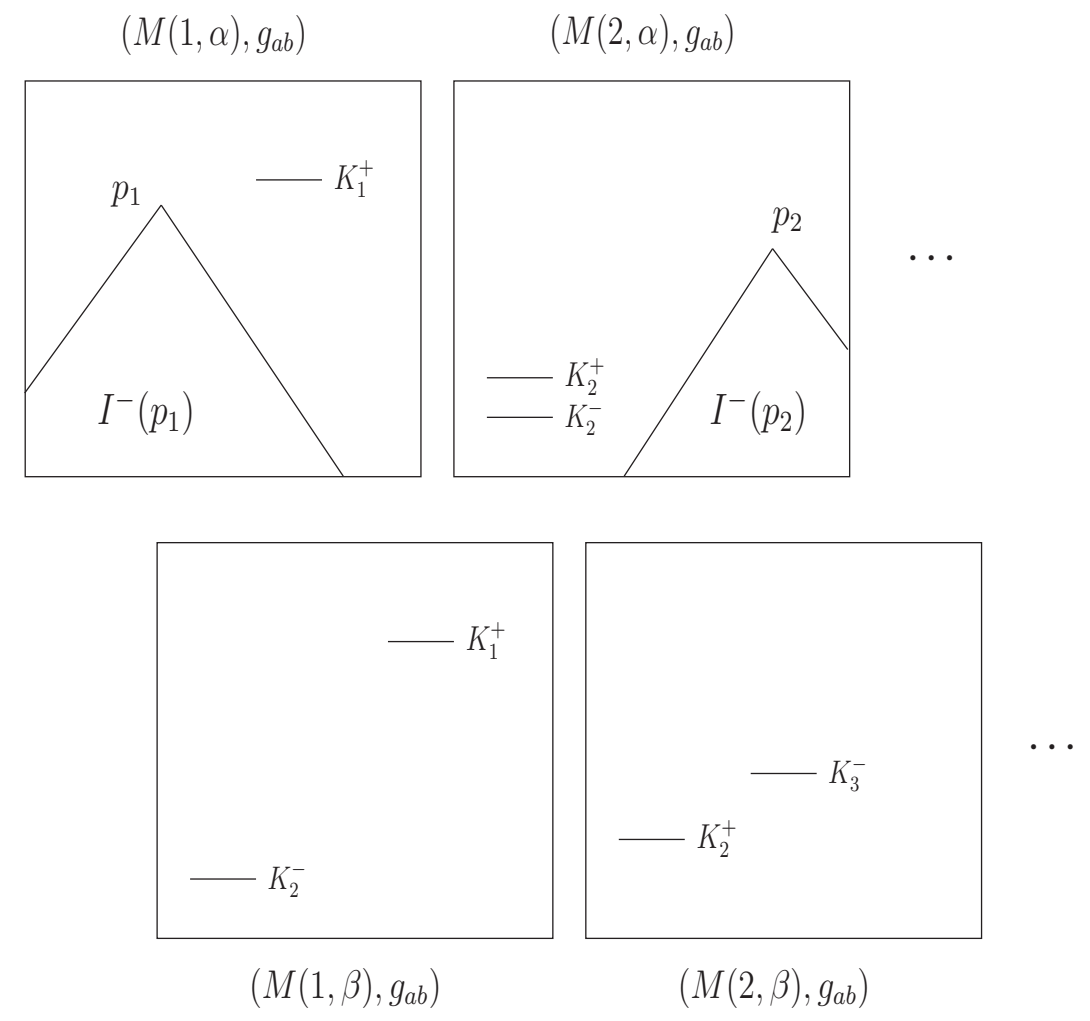

Fig. 2. The spacetime $\left(M^{\prime}, g_{a b}^{\prime}\right)$. Here, for all $i$, we identify the upper edge of $K_{i}^{+}$ in $\left(M(i, \alpha), g_{a b}\right)$ with the lower edge of $K_{i}^{+}$in $\left(M(i, \beta), g_{a b}\right)$ (excluding boundary points) and the lower edge of $K_{i}^{+}$in $\left(M(i, \alpha), g_{a b}\right)$ with the upper edge of $K_{i}^{+}$in $\left(M(i, \beta), g_{a b}\right)$. Similarly, we identify the upper edge of $K_{i+1}^{-}$in $\left(M(i, \beta), g_{a b}\right)$ with the lower edge of $K_{i+1}^{-}$in $\left(M(i+1, \alpha), g_{a b}\right)$ and vice versa.

To see that condition (i) holds, consider any point $q \in M$. Clearly, $q \in I^{-}\left(p_{i}\right)$ for some $i$. Because $I^{-}\left(p_{i}\right) \subset M(i, \alpha)$ one can always find a neighborhood $O$ of $q$ and an open set $O^{\prime} \subset M(i, \alpha) \subset M^{\prime}$ such that $O$ and $O^{\prime}$ are isometric. Next consider any point $q^{\prime} \in M^{\prime}$. By construction, $q^{\prime} \in M(i, \alpha)$ or $q^{\prime} \in M(i, \beta)$ for some $i$. But either way $q^{\prime} \in M$ (because $\left.M(i, \alpha), M(i, \beta) \subset M\right)$. So clearly, one can always find a neighborhood $O^{\prime}$ of $q^{\prime}$ and an open set $O$ on $M$ such that $O$ and $O^{\prime}$ are isometric. So the two spacetimes are locally isometric and therefore $\left(M^{\prime}, g_{a b}^{\prime}\right)$ satisfies the set $\mathfrak{C}$ of local conditions. So, condition (i) is satisfied.

To see that condition (ii) holds, consider any point $q \in M$. Clearly $I^{-}(q) \subset$ $I^{-}\left(p_{i}\right)$ for some $i$. But consider any $I^{-}\left(p_{i}\right)$. By construction, $I^{-}\left(p_{i}\right) \subset M(i, \alpha) \subset$ $M^{\prime}$. So there will always be an isometric counterpart to $I^{-}\left(p_{i}\right)$ in $M^{\prime}$. Thus, condition (ii) is satisfied and we are done. 


\section{Conclusion}

The theorem above seems to indicate that we cannot know the global structure of spacetime even if we make the unverifiable assumption, as do the cosmologists, that "the normal physical laws we determine in our spacetime vicinity are applicable at all other spacetime points." 11 In other words, there is a sense in which, even if we restrict our attention to "physically reasonable" spacetimes, we face a substantial epistemological predicament.

One may wonder about the role of matter fields in our discussion of observational indistinguishability. It could be argued, for example, that when these fields are taken into consideration, one may be able, from the observations of such fields, to distinguish between "observationally indistinguishable" spacetimes. But we suggest here that the "clothesline construction" utilized in our proof can rule out any objections of this kind. ${ }^{12}$ So, it seems that there is a robust sense in which the global structure of every spacetime is underdetermined.

\section{References}

Earman, J. (1993). Underdetermination, realism, and reason. In P. French, T. Uehling, \& H. Wettstein (Eds.), Midwest studies in philosophy Vol. XVIII (pp. 19-38). Chicago: University of Notre Dame Press.

Ellis, G. F. R. (1975). Cosmology and verifiability. Quarterly Journal of the Royal Astronomical Society, 16, 245-264.

Ellis, G. F. R. (1980). Limits to verification in cosmology. Annals of the New York Academy of Sciences, 336, 130-160.

Ellis, G. F. R. (2007). Issues in the philosophy of cosmology. In J. Butterfield \& J. Earman (Eds.), Handbook of the philosophy of physics (pp. 1183-1286). Oxford: Elsevier.

Geroch, R. (1977). Prediction in general relativity. In J. Earman, C. Glymour, \& J. Statchel (Eds.), Foundations of space-time theories, Minnesota studies in the philosophy of science Vol. VIII (pp. 81-93). Minneapolis: University of Minnesota Press.

Geroch, R., \& Horowitz, G. (1979). Global structure of spacetimes. In S. Hawking \& W. Isreal (Eds.), General relativity: An Einstein centenary survey (pp. 212-293). Cambridge: Cambridge University Press.

Glymour, C. (1972). Topology, cosmology, and convention. Synthese, 24, 195218.

\footnotetext{
${ }^{11}$ Ellis (1975, p. 246). See also Ellis (1980, 2007).

${ }^{12}$ See Malament (1977, p. 74-76) for details.
} 
Glymour, C. (1977). Indistinguishable space-times and the fundamental group. In J. Earman, C. Glymour, \& J. Statchel (Eds.), Foundations of space-time theories, Minnesota studies in the philosophy of science Vol. VIII (pp. 5060). Minneapolis: University of Minnesota Press.

Glymour, C. (1980). Theory and evidence. Princeton: Princeton University Press.

Hawking, S., \& Ellis, G. F. R. (1973). The large scale structure of space-time. Cambridge: Cambridge University Press.

Leplin, J. (1997). The underdetermination of total theories. Erkenntnis, 47 , 203-215.

Malament, D. (1977). Observationally indistinguishable space-times. In J. Earman, C. Glymour, \& J. Statchel (Eds.), Foundations of space-time theories, Minnesota studies in the philosophy of science Vol. VIII (pp. 61-80). Minneapolis: University of Minnesota Press.

Norton, J. (2008). Must evidence underdetermine theory? In M. Carrier, D. Howard, and J. Kourany (Eds.), The challenge of the social and the pressure of practice: Science and values revisited (pp. 17-44). Pittsburgh: University of Pittsburgh Press.

Sklar, L. (1985). Philosophy and spacetime physics. Berkeley: University of California Press.

Stanford, K. (2006). Exceeding our grasp: Science, history, and the problem of unconceived alternatives. Oxford: Oxford University Press.

van Fraassen, B. (1980). The scientific image. Oxford: Clarendon Press.

Wald, R. (1984). General relativity. Chicago: University of Chicago Press. 